\title{
Biliary Cystic Tumours with Mesenchymal Stroma
}

\author{
Andrejs Vanags, Maris Pavars, Peteris Prieditis, Ilze Strumfa, Arvids Irmejs, Janis Gardovskis \\ Riga Stradins University, Riga, Latvia
}

\begin{abstract}
Summary
Biliary cystadenoma and cystadenocarcinoma are rare cystic liver tumours arising from the bile ducts proximal to the hilum of the liver. The tumours have unique structure with ovarian-type mesenchymal stroma, leading to challenging considerations about their pathogenesis. The differential diagnosis is wide and further complicated by the lack of awareness about these neoplastic processes due to the rarity of the disease. Radical surgical treatment can be recommended whenever possible as it can result in prolonged survival.
\end{abstract}

Key words: biliary cystadenoma; biliary cystadenocarcinoma; mesenchymal stroma.

\section{INTRODUCTION}

Biliary cystadenocarcinoma (BCA) is an unusual cystic neoplasm of intrahepatic bile ducts. It is defined by cystic structure, composed of papillary epithelium with clearcut signs of malignancy: cellular atypia, particularly nuclear polymorphism; mitotic activity and invasive growth. The benign counterpart of BCA is the biliary cystadenoma (BA), lacking the malignant features (4). The cystic biliary tumours (CBT) represent rare entities, with incidence of BCA approximately $1 / 10$ million patients and of BA 1/20 000-1/100 000 (12).

CBT arise from bile ducts proximal to the hilum of the liver and share the cystic structure and presence of peculiar ovarian-type mesenchymal stroma with mucinous cystic tumours of the pancreas and retroperitoneum, leading to the hypothesis that ectopic ovarian stroma during embryogenesis can become incorporated along the biliary tree, in the pancreas and retroperitoneal space and cause the proliferation of the adjacent epithelium by production of hormones and growth factors (28). Origin from intrahepatic peribiliary glands (22), ectopic rests of primitive foregut sequestered in the liver (31) or pluripotential stem cells (3) has also been hypothesised. BCA without mesenchymal stroma more frequently arises in males and carries worse prognosis (22). There is evidence showing that at least some cases of BCA originate from BA. These data include the age difference between BCA and BA patients (18) as well as morphologic findings of malignant transformation in a lesion with focally innocuous structure (6).

BCA mostly develops intrahepatically $(83 \%)$; however, origin from extrahepatic bile ducts (13\%) or gall bladder $(0.02 \%)$ is possible $(18)$. BCA spreads within the liver as well as towards regional lymph nodes in the hepatoduodenal ligament. Distal metastases may develop in the lungs, the pleura or the peritoneum. TNM classification of liver tumours is applied for staging (4).

As was noted, the incidence of BCA is as low as one per 10 million patients (12). Due to the rarity of the disease, mostly case reports or small retrospective series are published, including 222 cases in the world medical literature, 1974-2008. Three (0.4\%) cases of BCA were identified among 740 patients who underwent hepatic resection at the University Health Sciences Center of Colorado and the University of Pittsburgh Medical Center, 1964-1991 (14). In the same group, the frequency of BCA among the resected cystic lesions was $6.8 \%(3 / 44)$. BCA comprised $0.18 \%$ of all liver tumours registered by Japanese Liver Cancer Study Group (22). It has been estimated that $5-11 \%$ of patients with large liver cysts presenting for treatment or diagnosed in autopsy have BA (23), the benign counterpart of BCA and possible precursor of it. Other authors have cited that $5 \%$ of all hepatic cysts represent either benign or malignant CBT (17). However, such high frequency of CBT among all liver cysts would correspond to prevalence that is higher than of cholangiocarcinoma while even BA is rarer and BCA comprises $5.3-25 \%$ of both CBT (23). It has to be concluded that the early data are biased both by patient selection and also by diagnostic errors due to inferior material and methodology.

The average age of patients developing BCA is 50-60 years (4). The patients with benign CBT are younger than the patients with malignant CBT: mean age 40.6 (range 30-51) vs. 51.3 (range 41-63) years (18). Other authors have found that the mean age of BA patients is even 17 years less than for $\operatorname{BCA}(2,25)$. CBT are more common in women: $80-100 \%$ of BA and $63-71.4 \%$ of BCA are described in female (18).

The most frequent clinical manifestation of CBT is the abdominal pain $(23,30)$, but abdominal distension, nausea (23), jaundice, cholangitis, tumour rupture, haemorrhage, compression of the portal or caval veins with possible subsequent ascites (30), hemobilia (14) and mucobilia (9) may occur. The most frequent physical finding is palpable abdominal mass (30); low location of lower liver margin can be found by percussion (31). The symptoms are non-specific and attributable to mass lesion as pain, tumour rupture $(31)$, bleeding $(11,14)$, obstructive jaundice and ascites due to the compression of caval or hepatic vein (31) have been observed in the case of benign cystic lesions. 
The development of CBT can last several years $(2,23)$. A case of biliary cystadenocarcinoma followed up as benign cystadenoma for 10 years has been described. The tumour still was resectable and the patient was free of any detectable recurrence for at least 4 years after the operation (13). Malignant transformation was observed in intrahepatic cystadenoma followed by serial biopsies (26). The long course is in accordance with the low grade of malignancy and gradual development of tumour through stages of increased epithelial proliferation, dysplasia, in situ cancer and, finally, invasive cancer. Thus, presence of cystic hepatic mass for several years does not exclude the possibility of malignant tumour and the need for careful follow-up if the cyst is not removed by operation.

Laboratory findings, including liver enzyme and bilirubin levels in blood, are abnormal if biliary tree is compressed (30). The serum carbohydrate antigen (CA19-9) and carcinoembryonic antigen (CEA) in BCA patients can be within the normal range (18); an abnormal level is not indicative of malignancy (31). Elevated levels of CEA and CA19-9 in the cyst fluid are reported in CBT but not in simple cysts (17).

The size of CBT varies from $1.5-30 \mathrm{~cm}$ (17) and is not helpful in the differential diagnostics between simple hepatic cyst and CBT as well as between BA and BCA. Grossly, BCA represents single multicystic lesion with internal mural nodules (fig. 1). Communication with the biliary tree has been reported by some and denied by other authors (18). Morphologically, BCA has cystic and papillary architecture (fig. 2). The cuboidal and non-ciliated columnar cells show atypia; invasion is the hallmark of malignant growth. The presence of mitoses is the third morphological diagnostic criterion of malignancy in a CBT (4). Oncocytic differentiation is described in at least 3 occasions (1). The BA is characterised by either mucinous or serous benign epithelium. The mesenchymal, ovarian-type stroma is present in benign and most malignant CBT.

Immunohistochemistry is of great importance in the differential diagnosis between primary and metastatic liver malignancies. There is lack of wide immunohistochemical studies of BCA; however, some case reports are available. Gradually increasing proliferative activity as characterised by Ki-67 as well as increasing p 53 protein expression from adenoma to carcinoma was shown in BCA without ovarian-type stroma (7). Expression of cytokeratin (CK) 7 and absence of CK20, CEA, alpha-fetoprotein, calretinin, CD31 and chromogranin is described in biliary cystadenocarcinoma with oncocytic differentiation (1). However, presence of CK20, although highly typical for colorectal cancer, has been described also in cholangiocarcinoma, especially non-peripheral (20). It might be expected in BCA with growing awareness about this entity and, indeed, was reported by our group (24).

Although BCA is rare, liver cysts are frequent, being present in $2.5 \%$ of the population (16) or even $18 \%$ of asymptomatic patients (23). Because of the differences in the treatment, this differential diagnosis is of great importance. CA19-9 and CEA levels are not diagnostic for this purpose $(18,30)$. Fine needle aspiration or core biopsy is unlikely to yield sufficient tissue in case of simple cyst; it is also not suitable for the diagnostics of focal malignancy and rarely can lead to peritoneal carcinomatosis (15). Therefore radiological diagnostics, especially computed tomography (CT), is essential in the diagnostics (23). Transabdominal ultrasonography also is frequently used (23).

CBT characteristically are solitary large multilocular lesions (fig. 3). Presence of internal septations allows excluding a simple cyst. Vascularity of septa is characteristic for CBT (18) and is considered by some specialists to be more reliable in distinguishing BA from cyst than the simple presence of septations (23). Biliary cystadenoma is characterised by smooth and thin internal septa, but presence of enhanced mural nodules (18) in the outer wall or septa is the most important sign of malignancy. Calcification is not frequent but has been found specific for malignancy by some (18) but not all (16) authors as far as CBT are concerned. Size, number of septations or location of the neoplasm does not help to differentiate between benign or malignant CBT (18). Some authors have postulated that preoperative differentiation between BA and BCA by CT or magnetic resonance imaging (MRI) is not possible therefore liver resection should be performed for all CBT (17). This assumption is based on the experience that internal papillae with arterial enhancement may be present in both tumours so that CT and MRI yield overlapping data. Cholangiography is strongly advised by some specialists in order to detect communication between the cystic tumour and bile ducts $(17,23)$. However, contrary opinions are reported based on the finding that the communication with bile ducts can be appropriately treated during operation (23) without intraoperative cholangiography. However, cholangiography is described as the most accurate method for the diagnosis of extrahepatic biliary cystadenomas (2).

Cystic liver lesions, entering the differential diagnosis of BCA, include developmental, neoplastic, inflammatory and traumatic lesions as simple bile duct cyst, polycystic liver disease, biliary hamartoma, cystically degenerated cases of other primary or metastatic liver tumours, abscesses, hydatid cyst, extrapancreatic pseudocyst, hematoma and biloma $(10,16)$. The short characteristics of these lesions are following. Simple cysts are asymptomatic, solitary or multiple, possessing thin wall. They appear in the CT and MRI as non-enhancing, well circumscribed homogeneous lesions, filled with fluid. Multiple hepatic cysts can be the manifestation of autosomal dominant polycystic liver disease. In these patients, complications as intracystic haemorrhage are more common and can be evaluated by MRI. Bile duct hamartomas typically are multiple, measure less than $1.5 \mathrm{~cm}$ and are less smoothly contoured than simple cysts. The autosomal recessive Caroli disease represents the congenital cavernous ectasia of the biliary ducts, frequently complicated by stone formation. Communication with the biliary tract is diagnostically 
important as well as the "central dot" sign by CT and bridges across the saccular dilatations by MRI. Both the aforementioned features result from the presence of portal vein branches within remaining connective tissue strands surrounded by dilated bile ducts. Embryonal sarcoma, rare and usually solid malignant tumour of adolescence, could seem cystic on CT and MRI due to myxoid stroma. The age and mostly solid appearance help to distinguish this tumour from BCT. Metastases can undergo cystic degeneration due to either necrosis or mucus secretion. Necrotic metastases by CT and MRI are revealed as multiple lesions with strong enhancement of peripheral viable tissue but irregular border. Mucinous metastases mostly are caused by disseminated colorectal or ovarian cancer; the latter can be recognised as mostly peripheral nodules due to transperitoneal implantation metastases in the capsule rather than intraparenchymal hematogenous spread (16). The multilayered wall and presence of multiple small hypoattenuating daughter cysts with thin eggshell calcifications allow recognising echinococcosis; if doubt exists, serologic assay must be applied $(17,19)$. Abscesses characteristically develop in the setting of sepsis or ascending cholangitis. Presence of air points towards gas-forming microbe. Acute abscess represents a cluster of small lesions that later converges into unilocular cystic lesion. Thick-walled lesion with enhancing wall develops and the "double target" sign can be observed due to increased peripheral rim enhancement secondary to increased capillary permeability. However, invasive malignancy can cause the same reaction (16). Presence of mobile debris at ultrasonography is characteristic of abscess (17). Biloma at CT and MRI appears as a well-defined cystic structure without septa, calcifications or pseudocapsule; history of trauma or operation also is helpful in order to suspect biloma or hematoma (16). In difficult cases, the diagnosis is made by histology (21).

To achieve long term cure, the ultimate goal of the surgical treatment of CBT is to remove all the neoplastic tissue. Treatment of CBT by marsupialization, internal Roux-en-Y drainage, aspiration, sclerosing or partial resection has high rate of complications - tumour recurrence and progression or sepsis. On the contrary, complete removal of the tumour can lead to prolonged survival $(14,29)$. However, there are different opinions towards the extent of operation in order to ensure radicality.

Definite operative intervention for CBT as tried in the Vanderbilt University School of Medicine on 2005, has included hepatic resection in 12/19 patients and enucleation in $6 / 19$ patients. No perioperative deaths have occurred among these patients. Complications were limited to 1 biloma and 2 intraabdominal abscesses treated by percutaneous drainage; biliary leak treated by stenting, as well as non-surgical complications like atrial fibrillation, acute lung injury and pleural effusion. Fenestration and fulguration has been used in 2 patients with large cystic liver lesions. In one of these cases, laparoscopic fenestration and fulguration has been used as the main treatment. A satellite lesion adjacent to resected larger BCA mass also was fulgurated and the patient was free of disease 2142 days after operation (23). In the whole group of radically treated patients no recurrences have been observed after mean follow-up of 3.5 years with standard deviation 4.2 years (23).

Data confirming the safety of hepatic resection in specialized institutions are not confined to recent progress. For instance, results in patients operated between 1964 and 1991 are described (14). The relevant medical team has performed 44 hepatic resections for cystic liver lesions of different nature, having 1 operative death in a Jehovah's Witness and 9 major complications with complete recovery in all but 2 cases due to progression and spread of malignant tumour. The complications included 3 subphrenic abscesses, subarachnoid haemorrhage and 2 re-explorations for bleeding and pulmonary embolism. Authors suggested that resection due to its safety and efficacy is even better than aspiration, sclerotherapy, internal drainage, marsupialisation and fenestration for congenital hepatic cyst. The fact that none of the CBT was diagnosed correctly before the curative operation adds justification to recommend more aggressive surgical treatment for cystic liver lesion (14). Other groups have suggested that preoperative diagnosis of CBT can be reached in 95\% although many patients have had wrong prior diagnosis and even operative treatment that was insufficient due to erroneous diagnosis and thus lead to recurrence (23). High level of awareness is a necessity in order to diagnose CBT.

In summary, enucleation or radical excision can be recommended for $\mathrm{BA}$, but lobectomy or radical resection is recommended for BCA (18). In multiseptated cyst without definite preoperative diagnosis, the rate of malignancy is as high as $25-45 \%$, therefore enucleation or complete resection is indicated (23).

Systemic chemotherapy including 5FU has been reported effective in a single patient with recurrence of BCA 41 month after surgery (8). Hepatic arterial infusion of cisplatin has been effective in another patient, decreasing tumour size from 12 to $2 \mathrm{~cm}$ in diameter (5).

If $\mathrm{BA}$ has been completely removed, the prognosis is excellent; otherwise malignant change may occur (26). The prognosis of BCA is significantly better than for other primary malignant liver tumours. Prolonged survival can be expected (14), even 156 months after radical surgery (29).

\section{CONCLUSIONS}

1. Increased awareness of biliary cystadenocarcinoma and cystadenoma could be helpful in recognising these rare primary intrahepatic tumours.

2. Due to the differences in the treatment and prognosis of different cystic liver lesions, as well as to lower informativity of other diagnostic methods, the radiological examination, especially $\mathrm{CT}$, is important in the preoperative diagnostics. Presence of internal vascularised septa is characteristic for cystic biliary tumours but presence of mural nodules or calcifications should cause suspicion of malignancy. 
3. The final diagnosis depends on histological findings. In a cystic liver tumour featuring ovarian-type stroma, the presence of nuclear atypia and mitoses in tumour cells and invasive growth proves biliary cystadenocarcinoma. Immunohistochemistry should be used to distinguish between metastasis and primary origin of the tumour from biliary epithelium. Staging by pTNM of liver tumours should be applied. Biliary cystadenoma is characterised by cystic structure, mesenchymal stroma and benign epithelium.

4. To achieve long term cure, the ultimate goal of the surgical treatment of biliary cystic neoplasms is to remove all the neoplastic tissue.

5. Appropriate surgical treatment of biliary cystadenocarcinoma may ensure prolonged survival.

6. The workup of cystic biliary tumour thus can be summarised as radiologic diagnostics including CT but possibly not limited by it; surgery and final pathological diagnosis. It represents a manageable challenge for modern medical team.

\section{Conflict of interest: None}

\section{REFERENCES}

1. Bardin RL, Trupiano JK, Howerton RM, Geisinger KR. Oncocytic biliary cystadenocarcinoma: a case report and review of the literature // Arch Pathol Lab Med, 2004; 128(2):25 - 28

2. Davies W, Chow M, Nagorney D. Extrahepatic biliary cystadenomas and cystadenocarcinoma // Ann Surg, 1995; 222(5):619-625

3. D'Errico A, Deleonardi G, Fiorentino M, Scoazek JY, Grigioni WF. Diagnostic implications of albumin messenger RNA detection and cytokeratin pattern in benign hepatic lesions and biliary cystadenocarcinoma // Diagn Mol Pathol, 1998; $76: 289-294$

4. Hamilton SR, Aaltonen LA (eds.) Pathology and genetics. Tumours of the digestive system. Lyon: IARC Press; 2000; 1-314

5. Hanazaki K, Yoshizawa K, Mori H. Hepatic arterial infusion chemotherapy of cisplatin for biliary cystadenocarcinoma // Hepatogastroenterology, $1999 ; 46(25): 462-464$

6. Ishak KG, Willis GW, Cummins SD, Bullock AA. Biliary cystadenoma and cystadenocarcinoma: report of 14 cases and review of the literature // Cancer, 1977; 38:322 - 338

7. Ishibashi $\mathrm{Y}$, Ojima H, Hiraoka N, Sano T, Kosuge $\mathrm{T}$, Kanai Y. Invasive biliary cystic tumour without ovarian-like stroma // Pathol Int, 2007; 57(12): $794-798$

8. Iyama S, Takahashi $\mathrm{Y}$, Shintani N, Fujikawa K, Ohkubo S, Sato Y, Sato T, Sato Y, Ohnuma K, Niitsu Y. [A case of recurrent biliary cystadenocarcinoma successfully treated with 5FU/CDDP systemic chemotherapy] // Nippon Shokakibyo Gakkai Zasshi, 2006; 103(10):1163 - 1168. [Pubmed abstract PMID 17023760]
9. Jan YY, Chen MF, Chen TJ. [Cholangiocarcinoma with mucobilia] // J Formos Med Assoc, 1994; 93. Suppl 3: S149 - 155. [Pubmed abstract PMID7606173]

10. Karahan OI, Kahriman G, Soyuer I, Ok E. Hepatic von Meyenburg complex simulating biliary cystadenocarcinoma // Clin Imaging, 2007; 31(1): $50-53$

11. Kitajima Y, Okayama Y, Hirai M, Hayashi K, Imai H, Okamoto T, Aoki S, Akita S, Gotoh K, Ohara H, Nomura T, Joh T, Yokoyama Y, Itoh M. Intracystic hemorhage of a simple liver cyst mimicking a biliary cystadenocarcinoma. J Gastroenterol, 2003; 38(2):190 - 193

12. Koffron A, Rao S, Ferrario M, Abecassis $M$. Intrahepatic biliary cystadenoma: role of cyst fluid analysis and surgical management in the laparoscopic era // Surgery, 2004; 136:926 - 936

13. Kubota E, Katsumi K, Iida M, Kishimoto A, Ban Y, Nakata K, Takahashi N, Kobayashi K, Andoh K, Takamatsu S, Joh T. Biliary cystadenocarcinoma, followed up as benign cystadenoma for 10 years // J Gastroenterol, 2003; 38(3):278 - 282

14. Madariaga JR, Iwatsuki S, Starzi TE, Todo S, Selby $\mathrm{R}$, Zetti G. Hepatic resection for cystic lesions of the liver // Ann Surg, 1993; 218(5):610 - 614

15. Manouras A., Markogiannis H, Lagoudianakis E, Katergiannakis V. Biliary cystadenoma with mesenchymal stroma: report of the case and review of the literature // World J Gastroenterol, 2006; 12(37):6062 - 6069

16. Mortele KJ, Ros PR. Cystic focal liver lesions in the adult: differential CT and MR imaging features // RadioGraphics 2001; 21:895 - 910

17. Poggio P, Buonocore M. Cystic tumours of the liver: A practical approach // World J Gastroenterol, 2008; 14(23):3616 - 3620

18. Pojchamarnwiputh S, Na Chiangmai W, Chotirosniramit A, Lertprasertsuke N. Computed tomography of biliary cystadenoma and biliary cystadenocarcinoma // Singapore Med J, 2008; 49(5):392 - 396

19. Proietti S, Abdelmoumene A, Genevay M, Denys A. Ecchinococcal Cyst // RadioGraphics, 2004; 24: $861-865$

20. Rullier A, Le Bail B, Fawaz R, Blanc JF, Saric J, Bioulac-Sage P. Cytokeratin 7 and 20 expression in cholangiocarcinomas varies along the biliary tree but still differs from that in colorectal carcinoma metastasis // Am J Surg Pathol, 2000; 24(6): $870-876$

21. Shrikhande S, Kleef J, Adyanthaya K, Zimmermann A, Shrikhande V. Management of hepatobiliary cystadenocarcinoma // Dig Surg, 2003; 20:60 - 63

22. Sudo Y, Harada K, Tsuneyama K, Katayanagi K, Zen Y, Nakanuma Y. Oncocytic biliary cystadenocarcinoma is a form of intraductal papillary neoplasm of the liver // Mod Pathol, 2001; 14(12):1304 - 1309

23. Thomas KT, Welch D, Trueblood A, Sulur P, Wise P, Gorden L, Chari RS, Wright JK, Washington 
K, Pinson CW. Effective treatment of biliary cystadenoma // Ann Surg, 2005; 241:769 - 775

24. Vanags A, Pavars M, Prieditis P, Strumfa I, Irmejs A, Gardovskis J. Biliary cystadenocarcinoma: a case study of a rare tumour // Acta Chirurgica Latviensis, 2008; 8:90-93.

25. Wheeler DA, Edmondson HA. Cystadenoma with mesenchymal stroma (CMS) in the liver and bile ducts // Cancer, 1985; 56(6):1434- 1435

26. Woods G. Biliary cystadenoma: case report of hepatic malignancy originating in benign cystadenoma // Cancer, 1981; 47:2936 - 2940

27. Yu FC, Chen JH, Yang KC, Wu CC, Chou YY. Hepatobiliary cystadenoma: a report of two cases // J Gastrointestin Liver Dis, 2008:17(2):203 - 206

28. Zamboni G, Scarpa A, Bogina G, Iacono C, Bassi C, Talamini, Sessa F, Capella C, Solcia E, Rickaert F, Mariuzzi GM, Klopel G. Mucinous cystic tumors of the pancreas: clinicopathologic features, prognosis, and relationship to other mucinous cystic tumors // Am J Surg Pathol, 1999; 23:410 - 422

29. Zen Y, Fujii T, Itatsu K, Nakamura K, Konishi F, Masuda S, Mitsui T, Asada Y, Miura S, Miyayama S, Uehara $\mathrm{T}$, Katsuyama $\mathrm{T}$, Ohta $\mathrm{T}$, Minato $\mathrm{H}$, Nakanuma Y. Biliary cystic tumors with bile duct communication: a cystic variant of intraductal papillary neoplasm of the bile duct // Mod Pathol, 2006; 19:1243 - 1254

30. Zhang M, Yu J, Yan S, Zheng SS. Cystadenocarcinoma of the liver: a case report // Hepatobiliary Pancreat Dis Int, 2005; 4(3):464 - 467

31. Zhou JP, Dong M, Zhang Y, Kong FM, Guo KJ, Tian YL. Giant mucinous biliary cystadenoma: a case report // Hepatobiliary Pancreat Dis Int, 2007; 6(1):101- 103

\section{Address:}

Andrejs Vanags,

Hereditary Cancer Institute,

Riga Stradins University,

Dzirciema Street 16, LV 1007,

Riga, Latvia

e-mail: vanags314@inbox.lv

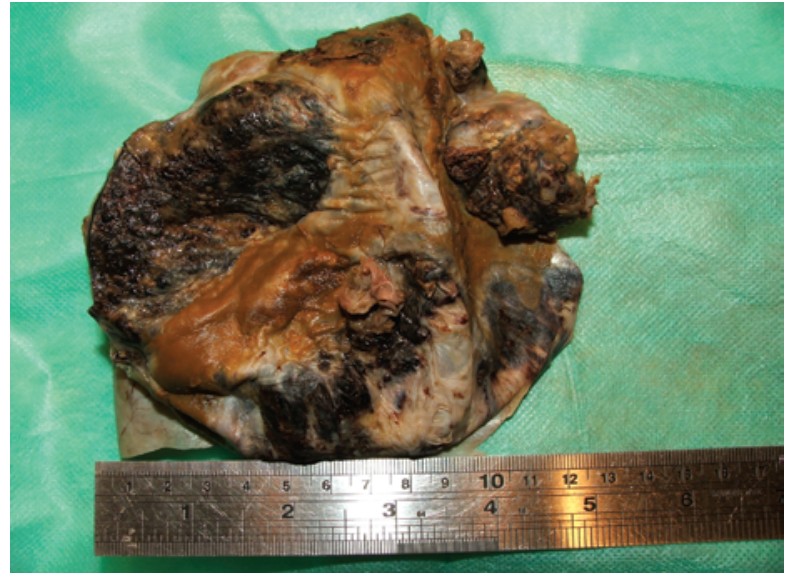

Fig. 1. Gross view of the inner surface of biliary cystadenocarcinoma in the formalin-fixed operation material. Note the marked, irregular nodularity

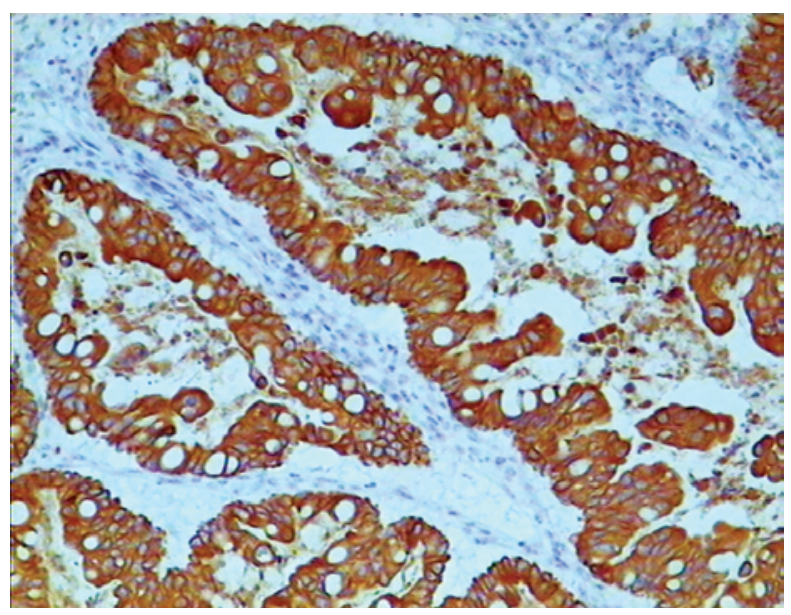

Fig. 2. Cystic and papillary architecture of biliary cystadenocarcinoma. Immunoperoxidase, anticytokeratin 7 , original magnification 100x

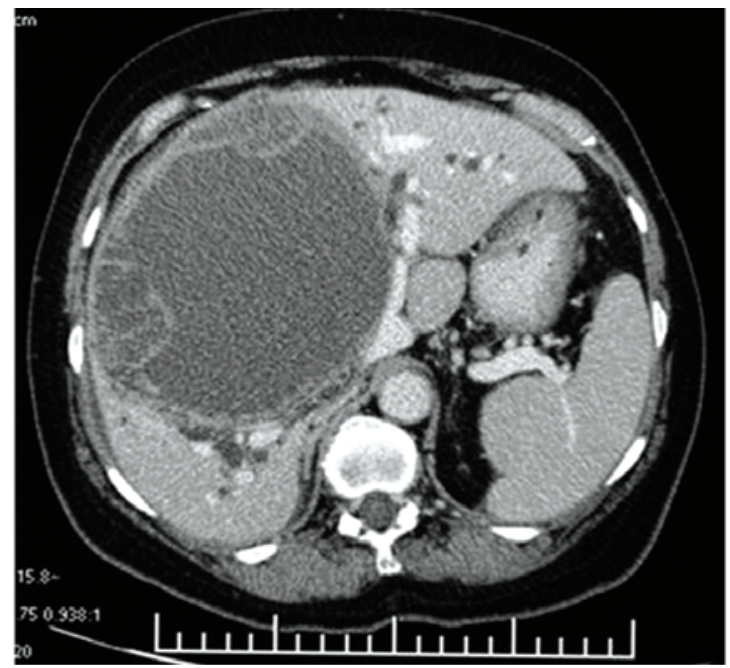

Fig. 3. Abdominal computed tomography scan in case of biliary cystadenocarcinoma 